\title{
On reachable sets for positive linear systems under constrained exogenous inputs *
}

\author{
Baozhu Du ${ }^{\mathrm{a}}$, James Lam ${ }^{\mathrm{b}}$, Zhan Shu ${ }^{\mathrm{c}}$, Yong Chen ${ }^{\mathrm{b}}$ \\ ${ }^{a}$ School of Automation, Nanjing University of Science and Technology, Nanjing, P. R. China. \\ ${ }^{\mathrm{b}}$ Department of Mechanical Engineering, University of Hong Kong, Pokfulam Road, Hong Kong. \\ ${ }^{\mathrm{c}}$ Electro mechanical engineering group, Faculty of engineering and the environment, University of Southampton, Highfield \\ Campus, Southampton SO17 1BJ, United Kingdom.
}

\begin{abstract}
This paper focuses on positive linear time-invariant systems with constant coefficients and specific exogenous disturbance. The problem of finding a hyper-pyramid to bound the set of the states that are reachable from the origin in the Euclidean space is addressed, subject to inputs whose $(1,1)$-norm or $(\infty, 1)$-norm is bounded by a prescribed constant. The Lyapunov approach is applied and a bounding hyper-pyramid is obtained by solving a set of inequalities. Iterative procedures (with an adjustable parameter) for reducing the hyper-volume of the bounding hyper-pyramid for the reachable set are proposed.
\end{abstract}

Key words: Hyper-pyramid; Linear programming; Linear system; Positive linear system; Reachable set.

\section{Introduction}

When an admissible control signal of a linear dynamic system is constrained in some way, the transfer of the system state from the origin to an arbitrary terminal state is generally not possible. Under some input constraints, the collection of all possible states to which the system can be transferred from the origin is referred to as the reachable set. The bounding of reachable states was first considered for linear systems in the late 1960s in the context of state estimation and it has later received a lot of attention in parameter estimation (see [8] and references therein). For linear systems, an ellipsoidal bound of the reachable set is often used to contain all the reachable states under zero initial conditions [5], [11], [12], [17], [18], [21], [23], [27], [28]. The idea may also be used for solving the peak-to-peak minimization problem [1] or control problems with saturating actuators [15], [22]. A linear matrix inequality (LMI) solution to the reachable set bounding problem was given in [2] via the Lyapunov approach. However, to the best of the authors' knowledge, no related work has been

\footnotetext{
* This paper was not presented at any IFAC meeting. Corresponding author Baozhu Du.

Email addresses: dubaozhu@gmail.com (Baozhu Du), jlam@hku.hk (James Lam), hustd8@gmail.com (Zhan Shu), cyong.hit@gmail.com (Yong Chen).
}

devoted to the reachable set bounding problem for positive systems. The novelty of the present work is that we derive, for the first time, a hyper-pyramidal bound on the reachable set of positive linear system under various types of norm-bounded disturbances.

On the other hand, a quantitative treatment of the performance and robustness of control systems requires the introduction of appropriate signal and system norms, which measure the magnitudes of the involved signals and system operators. As discussed in [6], some frequently used performance measures such as the $H_{\infty}$ or $L_{2}$ $L_{\infty}$ norms are based on the $L_{2}$ signal space, which are not very natural, in some situations, to describe the features of practical systems with positivity. On the other hand, the 1-norm of a vector-valued signal could provide a useful description for positive systems which measures the size of the input and/or output signals by summing the quantities of the non-negative components at a given time [7], and the $L_{1}$-norm measures the accumulation of all the components over time [25], which are more appropriate, for instance, when they represent the amount of material or the number of animals in a species. Thus, the performance of positive systems can be well evaluated based on the $L_{1}$-gain (that is, the induced norm of $L_{1}$ input and $L_{1}$ output). Naturally, the linear Lyapunov function can be applied as a valid candidate for stability analysis and controller synthesis of positive systems. By 


\section{CONFIDENTIAL. Limited circulation. For review only Automatica submission 15-1403.3}

using the linear Lyapunov approach, stability analysis, $L_{1}$-gain performance analysis and control design have been discussed for positive continuous-time linear systems [3], [13], [24] and positive continuous-time switched systems with delays [14], [19], [20]. In this paper, two types of admissible input signals are considered based on 1 -norm and/or $\infty$-norm. Under such specific classes of inputs, reachable set bounding and controller synthesis in the Euclidean space with hyper-pyramids are developed for positive linear systems.

The remaining parts of this article are organized as follows. In Section 2, preliminaries are presented for positive continuous-time systems. The input sets and the corresponding reachable sets are defined and their properties are briefly discussed in Section 3. Based on the characterizations of two classes of exogenous inputs, sufficient conditions are derived to find a hyper-pyramid that bounds the set of the states which are reachable from the origin. The problem can be tackled by finding an admissible positive vector subject to inequality constraints, and thus two iterative schemes are presented to construct a bounding hyper-pyramid for the system reachable set. The state-feedback synthesis problem is considered in Section 4 such that the closed-loop system is positive and its reachable set can be restrained within a certain hyper-pyramid.

\section{Notations:}

$\mathbb{N}_{+}, \mathbb{R} \quad$ set of positive integers, set of real numbers

$\mathbb{R}^{n} \quad$ set of $n$-dimensional real vectors

$\mathbb{R}^{m \times n} \quad$ set of $m \times n$ real matrieces

$\overline{\mathbb{R}}_{+}^{n}, \mathbb{R}_{+}^{n}$ nonnegative and positive orthants of $\mathbb{R}^{n}$

$e_{i} \quad$ vector with 1 in $i$ th position and 0 elsewhere

$\mathbf{1}, I \quad$ vector $[1,1, \ldots, 1]^{T}$, identity matrix

$\lambda_{i}(A) \quad i$ th eigenvalue of matrix $A$

$A^{T} \quad$ transpose of $A$

$\|x(t)\|_{1} \quad \sum_{i=1}^{n}\left|x_{i}(t)\right|, x(t)=\left[x_{1}(t), \ldots, x_{n}(t)\right]^{T} \in \mathbb{R}^{n}$

$\|x(t)\|_{\infty} \max _{i=1}^{n}\left|x_{i}(t)\right|, x(t)=\left[x_{1}(t), \ldots, x_{n}(t)\right]^{T} \in \mathbb{R}^{n}$

$\|\omega\|_{1,1} \quad \int_{0}^{\infty}\|\omega(s)\|_{1} \mathrm{~d} s$ (called $L_{1}$ norm in [7])

$\|\omega\|_{\infty, 1} \operatorname{ess} \sup _{t \geq 0}\|\omega(t)\|_{1}$

Moreover, $x \geq \geq 0(x>>0)$ denotes every componen$\mathrm{t}$ of $x$ is nonnegative (positive) ( $x$ is called nonnegative (positive) $) ; A \geq \geq 0(A>>0)$ denotes every entry of matrix $A$ is nonnegative (positive) ( $A$ is called nonnegative (positive)). A set $\mathcal{P}$ in a linear vector space is convex if $\alpha x_{1}+(1-\alpha) x_{2} \in \mathcal{P}$, for all $x_{1}, x_{2} \in \mathcal{P}$ and $\alpha \in[0,1]$. Furthermore, $\mathcal{P}$ is a convex cone if it is convex and in addition $\alpha x \in \mathcal{P}$ for all $x \in \mathcal{P}$ and all $\alpha>0$. Vectors and matrices, if their dimensions are not explicitly stat- ed, are assumed to have compatible dimensions for algebraic operations.

\section{Mathematical Preliminaries}

Definition 1 [16] Let $\mathbb{V}$ is a linear vector space, $s_{k}$ : $\mathbb{V} \rightarrow \mathbb{R}$. The constraint $s_{k}(y) \geq 0$ is said to be regular if there exists $y^{*} \in \mathbb{V}$ such that $s_{k}\left(y^{*}\right)>0, k=1,2, \ldots, N$, $N \in \mathbb{N}_{+}$.

Lemma 1 [16] Let $s_{k}: \mathbb{R}^{m} \rightarrow \mathbb{R}, s_{k}(y)=g_{k}^{T} y+h_{k}$, for $k=0,1, \ldots, N$, be linear functionals defined in a linear vector space $\mathbb{R}^{m}$, where $g_{k} \in \mathbb{R}^{m}, h_{k} \in \mathbb{R}$, and $N \in \mathbb{N}_{+}$. If $s_{k}(y)$ is regular for $k=1,2, \ldots, N$, the following two conditions are equivalent.

$\left(S_{1}\right) s_{0}(y) \geq 0$, for all $y \in \mathbb{R}^{m}$ such that $s_{k}(y) \geq$ $0, k=1,2, \ldots, N$.

$\left(S_{2}\right)$ There exist scalars $\tau_{k} \geq 0, k=1,2, \ldots, N$ such that $s_{0}(y)-\sum_{k=1}^{N} \tau_{k} s_{k}(y) \geq 0, \quad \forall y \in \mathbb{R}^{m}$.

Lemma 1 is a linear version of the classical quadratic Sprocedure [10], [26]. It is a valid tool for verifying the non-negativity of a linear function $s_{0}(y)$ under a finite number of linear constraints $s_{k}(y) \geq 0(k=1,2, \ldots, N)$ since condition $\left(S_{2}\right)$, in general, is much simpler to verify than condition $\left(S_{1}\right)$.

Definition $2[9] A \in \mathbb{R}^{n \times n}$ is Metzler if its off-diagonal elements are nonnegative, that is, $A_{(i, j)} \geq 0, i, j=$ $1,2, \ldots, n, i \neq j$.

\section{Reachable Sets with Exogenous Inputs}

Consider positive linear dynamic systems described by the vector differential equation:

$$
\dot{x}(t)=A x(t)+B_{\omega} \omega(t)
$$

where $x(t) \in \overline{\mathbb{R}}_{+}^{n}, \omega(t) \in \overline{\mathbb{R}}_{+}^{m}$ are the system state and an exogenous input signal, respectively, $A \in \mathbb{R}^{n \times n}$ and $B_{\omega} \in \overline{\mathbb{R}}_{+}^{n \times m}$ are constant system matrices, $B_{\omega}$ is nonzero.

When the input $\omega(t)$ is taken into account, we try to provide a fundamental characterization on the reachable set of system (1). The problem of bounding the reachable set of a linear system within an ellipsoid of $\mathbb{R}^{n}$, which has center at the origin, arises in many fields [2]. However, since the reachable states of positive system (1) with $\omega(t)$ lie in the first orthant as $x(t) \geq 0$, the set containing the reachable set is a subset of $\mathbb{R}_{+}^{n}$. To pose such a problem precisely, we need to know how the subset is described and what bounding criterion should be used (volume, semi-major or semi-minor axis, for instance). 


\subsection{Estimation of Reachable Sets}

A hyper-pyramid $\mathcal{C}_{p}$ of the form, for a given $p \in \mathbb{R}_{+}^{n}$ :

$$
\mathcal{C}_{p}=\left\{\xi \in \overline{\mathbb{R}}_{+}^{n} \mid p^{T} \xi \leq 1\right\}
$$

will be considered in this paper for bounding the set of reachable states of system (1) under zero initial conditions. Such a hyper-pyramid is a subset of the positive orthant and it is convex (that is, the segment connecting two points of the hyper-pyramid belongs to the hyperpyramid itself). It is clear that all the system states $x(t)$ are contained in $\mathcal{C}_{p}$ if and only if $p^{T} x(t) \leq 1$. Next, characterization on the hyper-pyramid $\mathcal{C}_{p}$ will be established for two possible classes of the exogenous input signal $\omega(t)$ based on the $L_{1}$-norm and 1-norm.

Case (i): $\quad \omega \in \Omega_{1,1} \triangleq\left\{\omega \in \overline{\mathbb{R}}_{+}^{m} \mid\|\omega\|_{1,1} \leq 1\right\}$.

In this case, the disturbance $\omega(t)$ is considered to have $L_{1}$-norm no greater than unity.

Theorem 1 The reachable set of positive system $\left(A, B_{\omega}\right)$ with zero initial conditions and input $\omega \in \Omega_{1,1}$ is bounded by the hyper-pyramid in (2) with vector $p \in \mathbb{R}_{+}^{n}$ if

$$
A^{T} p \leq \leq 0 ; B_{\omega}^{T} p \leq \leq \mathbf{1}
$$

Proof 1 Construct a Lyapunov function $V(x(t))=$ $p^{T} x(t)$ with $p>>0$, then $V(x(0))=p^{T} x(0)=0$. The derivative of $V(x(t))$ along the solution of system (1) with respect to $t$ is given by $\mathrm{d} V(x(t)) / \mathrm{d} t=$ $p^{T}\left(A x(t)+B_{\omega} \omega(t)\right) \leq \mathbf{1}^{T} \omega(t)$, which follows from $x(t) \geq \geq 0, \omega(t) \geq \geq 0$ and inequalites (3). Integrating its both sides from 0 to $T$, we get $V(x(T))-V(x(0)) \leq$ $\int_{0}^{T} \mathbf{1}^{T} \omega(s) \mathrm{d} s \leq 1$ for every $T \geq 0$ and every input $\omega$ satisfying $\int_{0}^{\infty}\|\omega(s)\|_{1} \mathrm{~d} s \leq 1$. Thus, $V(x(T)) \leq 1$, that $i s, p^{T} x(T) \leq 1$ for all $T \geq 0$. In other words, the hyperpyramid $\mathcal{C}_{p}$ contains the reachable set of system $\left(A, B_{\omega}\right)$. The proof is complete.

Remark 1 A natural conclusion derived from Theorem 1 is that, when $\int_{0}^{\infty}\|\omega(s)\|_{1} \mathrm{~d} s \leq \gamma$ where $\gamma>0$, the reachable set of positive system (1) is bounded by the hyper-pyramid in (2) if there exists a vector $p \in \mathbb{R}_{+}^{n}$ satisfying $A^{T} p \leq \leq 0$ and $B_{\omega}^{T} p \leq \leq \frac{1}{\gamma} \mathbf{1}$.

It can be seen from Theorem 1 that the Lyapunov stability of system (1) is a necessary condition for its reachable states bounded by a hyper-pyramid, while not necessarily asymptotic stability. If system (1) is known to be asymptotically stable, a necessary condition for bounding the reachable set can be established in the following theorem.

Theorem 2 Suppose that positive system $\left(A, B_{\omega}\right)$ is asymptotically stable. If the reachable sets under zero initial conditions and $\omega \in \Omega_{1,1}$ are bounded by the hyperpyramid (2) for some $p \in \mathbb{R}_{+}^{n}$, then

$$
B_{\omega}^{T} p \leq \leq \mathbf{1}
$$

and there exists at least an $i, i=1,2, \ldots, n$, such that

$$
e_{i}^{T} A^{T} p \leq 0
$$

Proof 2 Take $w(t)$ as $e_{i} \delta(t-T)$ for some $T>0$, where $e_{i} \in \mathbb{R}^{n}, i=1,2, \ldots, n$, and $\delta(t-T)$ is a Dirac measure. It is evident that $e_{i} \delta(t-T) \in \Omega_{1,1}$ and the system solution can be calculated as $x(t)=e^{A t} \int_{0}^{t} e^{-A \tau} B_{w} e_{i} \delta(\tau-T) d \tau=$ $e^{A(t-T)} B_{w} e_{i}$. It follows from this and $p^{T} x \leq 1$ that $p^{T} x(T)=p^{T} B_{w} e_{i} \leq 1$, for $i=1,2, \ldots, n$. Therefore, (4) holds.

To prove (5), we integrate equation (1) from 0 to $\infty$, that is, $x(\infty)-x(0)=A \int_{0}^{\infty} x(\tau) d \tau+B_{w} \int_{0}^{\infty} w(\tau) d \tau$. Since the system is asymptotically stable and $w(\tau) \in$ $\Omega_{1,1}$, one has that $x(\infty)=0$. From this and $x(0)=0$, it folllows that $p^{T} A \int_{0}^{\infty} x(\tau) d \tau=-p^{T} B_{w} \int_{0}^{\infty} w(\tau) d \tau \leq$ 0 . With this and the nonnegativity of $x(t),(5)$ is obvious.

For an asymptotically stable positive system, if inequality (4) or (5) in Theorem 2 does not hold, we can conclude that the reachable set under zero initial conditions and $\omega \in \Omega_{1,1}$ cannot be bounded by the prescribed hyper-pyramid $\mathcal{C}_{p}$. This theorem can be used for selecting an appropriate vector $p$ and related joint design of a state-feedback controller gain and $p$.

Case (ii): $\quad \omega \in \Omega_{\infty, 1} \triangleq\left\{\omega \in \overline{\mathbb{R}}_{+}^{m} \mid\|\omega\|_{\infty, 1} \leq 1\right\}$.

In this case, the disturbance $\omega(t)$ is considered to have 1-norm no greater than unity.

Theorem 3 The reachable set of positive system $\left(A, B_{\omega}\right)$ with zero initial conditions and input $\omega \in \Omega_{\infty, 1}$ is bounded by the hyper-pyramid in (2) with vector $p \in \mathbb{R}_{+}^{n}$ if there exists a scalar $\alpha>0$ satisfying

$$
A^{T} p+\alpha p \leq \leq 0 ; B_{\omega}^{T} p \leq \leq \alpha \mathbf{1}
$$

Proof 3 Suppose that there exists a Lyapunov function $V(\xi)=p^{T} \xi$ with $p>>0$. The hyper-pyramid given by (2) contains the reachable set of the positive system $\left(A, B_{\omega}\right)$ if $\mathrm{d} V(x(t)) / \mathrm{d} t \leq 0$ for any $x(t), \omega(t)$ satisfying (1), $\|\omega(t)\|_{1} \leq 1$ and $V(x) \geq 1$, which is equivalent to

$$
p^{T}\left(A x(t)+B_{\omega} \omega(t)\right) \leq 0
$$

for any $x(t)$ and $\omega(t)$ satisfying $\mathbf{1}^{T} \omega(t) \leq 1$ and $p^{T} x(t) \geq$ 1. Based on Lemma 1, condition (7) holds if and only if there exist $\alpha \geq 0$ and $\beta \geq 0$ such that, for all $x(t) \geq \geq 0$ and $\omega(t) \geq \geq 0, p^{T}[A x(t)+$ 
$\left.B_{\omega} \omega(t)\right]+\alpha\left[p^{T} x(t)-1\right]+\beta\left[1-\mathbf{1}^{T} \omega(t)\right] \leq 0$, that is, $\left(p^{T} A+\alpha p^{T}\right) x(t)+\left(p^{T} B_{\omega}-\beta \mathbf{1}^{T}\right) \omega(t)-\alpha+\beta \leq 0$, or equivalently, for all $x(t) \geq \geq 0$ and $\omega(t) \geq \geq 0$,

$$
p^{T} A+\alpha p^{T} \leq \leq 0, p^{T} B_{\omega}-\beta \mathbf{1}^{T} \leq \leq 0, \beta-\alpha \leq 0 .
$$

Clearly, $\alpha \geq \beta$. Next, if (8) holds for some $\left(\beta_{0}, \alpha_{0}\right)$, it also holds for all $\beta \in\left[\beta_{0}, \alpha_{0}\right]$ as $p^{T} B_{\omega}-\beta \mathbf{1}^{T} \leq \leq p^{T} B_{\omega}-$ $\beta_{0} \mathbf{1}^{T} \leq \leq 0$. Therefore, it can be assumed, without loss of generality, that $\alpha=\beta$, and (8) can be rewritten as $p^{T} A+\alpha p^{T} \leq \leq 0, p^{T} B_{\omega} \leq \leq \alpha \mathbf{1}^{T}$, which is guaranteed by (6). The proof is complete.

Based on the result proposed in Theorem 1, an alternative technique called exponential time-weighting procedure [2] can also be used to derive Theorem 3. For a given scalar $\alpha>0$ and every $T>0$, positive system (1) can be rewritten as

$$
\dot{x}_{T}(t)=(A+\alpha I) x_{T}(t)+B_{\omega} \nu(t), x_{T}(0)=0
$$

where $x_{T}(t)=e^{\alpha(t-T)} x(t)$ and $\nu(t)=e^{\alpha(t-T)} \omega(t)$ are new exponentially time-weighted variables. System (9) is a positive linear system since $A+\alpha I$ is Metzler. It follows from $\|\omega(t)\|_{1} \leq 1$ that $\int_{0}^{T}\|\nu(\tau)\|_{1} d \tau=$ $\int_{0}^{T} e^{\alpha(\tau-T)}\|\omega(\tau)\|_{1} d \tau \leq \int_{0}^{T} e^{\alpha(\tau-T)} d \tau \leq 1 / \alpha$. According to Theorem 1 and Remark 1 , when the input $\nu(t)$ satisfying $\int_{0}^{\infty}\|\nu(\tau)\|_{1} d \tau \leq \frac{1}{\alpha}$, the hyper-pyramid $\mathcal{C}_{p}$ contains the reachable set of the states $x_{T}(t)$ of system (9) if

$$
(A+\alpha I)^{T} p \leq \leq 0, B_{\omega}^{T} p \leq \leq \alpha \mathbf{1}
$$

which is $(6)$. The fact that $x_{T}(T)$ satisfies $p^{T} x_{T}(T) \leq 1$ implies $p^{T} x(T) \leq 1$. Since inequality (10) is independent of $T$, the hyper-pyramid $\mathcal{C}_{p}$ thus contains the reachable set of system (1) when $\omega \in \Omega_{\infty, 1}$.

Remark 2 For positive linear system (1), a sufficient condition for determining whether a point, denoted by $x_{a} \in \mathbb{R}^{n}$, lies outside the reachable set or not is that there exists a hyper-pyramid bounding the reachable set which does not contain $x_{a}$. In other words, the fact that $x_{a}$ does not belong to the reachable set can be checked by verifying an inequality $p^{T} x_{a}>1(p>>0)$ together with (3) or (6) for different exogenous input signal $\omega \in \Omega_{1,1}$ or $\omega \in \Omega_{\infty, 1}$, respectively.

\subsection{Optimization of Hyper-Pyramids}

A geometric interpretation on a hyper-pyramid $\mathcal{C}_{p}=$ $\left\{\xi \in \overline{\mathbb{R}}_{+}^{n} \mid p^{T} \xi \leq 1\right\}$, with $p=\left[p_{1}, p_{2}, \ldots, p_{n}\right]^{T} \in \mathbb{R}_{+}^{n}$, is a structure formed by connecting the hyper-plane $\left\{\xi \in \overline{\mathbb{R}}_{+}^{n} \mid p^{T} \xi=1\right\}$ and $n$ coordinate planes in the first orthant (its $n+1$ vertices are, respectively, the origin and $1 / p_{i}$ units along the $i$ th axis, $\left.i=1,2, \ldots, n\right)$.
Its hyper-volume depends on the product of $1 / p_{i}$, $i=1,2, \ldots, n$, since the $n$-dimensional hyper-volume of any hyper-pyramid is $1 / n$ of the $(n-1)$-dimensional base hyper-area multiplying its perpendicular height [4]. Therefore, the hyper-volume of hyper-pyramid

$$
\left\{x(t) \in \overline{\mathbb{R}}_{+}^{n} \mid p^{T} x(t) \leq 1, x(t) \text { satisfies }(1), \omega \in \Omega_{1,1}\right\},
$$

or

$\left\{x(t) \in \overline{\mathbb{R}}_{+}^{n} \mid p^{T} x(t) \leq 1, x(t)\right.$ satisfies $\left.(1), \omega \in \Omega_{\infty, 1}\right\}$

is equal to $\frac{1}{n !} \prod_{i=1}^{n} \frac{1}{p_{i}}$. To find a feasible hyper-pyramid that contains the reachable set of the positive system with hyper-volume as small as possible, we can minimize the hyper-volume over hyper-pyramids by minimizing $-\sum_{i=1}^{n} \log p_{i}$ over the positive vector variable $p>>0$ subject to $(3)$, or (6).

Taking Case (i) as an example, based on Theorem 1, the optimization turns out to be a convex minimization problem with respect to the positive vector $p=$ $\left[p_{1}, p_{2}, \ldots, p_{n}\right]^{T}$ as follows.

\section{Optimization Problem MHVP (Minimal Hyper- Volume Problem):}

$$
\begin{array}{ll}
\operatorname{minimize} & -\sum_{i=1}^{n} \log p_{i} \\
\text { subject to } & A^{T} p \leq \leq 0, B^{T} p \leq \leq \mathbf{1},
\end{array}
$$

Remark 3 As for $\omega \in \Omega_{\infty, 1}$ in Case (ii), it should be emphasized that the selection of $\alpha$ in (6) may affect the optimum value of the converged value $-\sum_{i=1}^{n} \log p_{i}$. Indeed, the first inequality in (6) indicates that $A$ is a Hurwitz matrix and $A+\alpha I$ is a semistable matrix. Therefore, $\alpha$ has an upper bound given by the spectral abscissa, $\min _{i=1}^{n}\left|\lambda_{i}(A)\right|$, denoted as $\bar{\alpha}$, that is, $\alpha \in(0, \bar{\alpha}]$. Various search routines, such as fminsearch.m provided in MATLAB Optimization Toolbox, can be applied to find an optimal $\alpha$ in that interval.

Optimization Problem MHVP finds a hyper-pyramid with the smallest hyper-volume by minimizing the product of $1 / p_{i}$, which is the length of the edge along the $i$ th axis, $i=1,2, \ldots, n$. Denote the optimal hyper-pyramid derived from (11) as $\mathcal{C}_{p}^{0}$. In general, $1 / p_{i}$ does not always achieve its minimum simultaneously in Optimization Problem MHVP. From a different perspective, if we only minimize $1 / p_{1}$ in the objective function, an admissible hyper-pyramid (denoted by $\mathcal{C}_{p}^{1}$ ) is found via (11). In the same way, minimizing $1 / p_{2}$, another admissible hyper-pyramid (denoted by $\mathcal{C}_{p}^{2}$ ) is then obtained, and the $n^{\text {th }}$ hyper-pyramid $\mathcal{C}_{p}^{n}$ will be obtained by minimizing $1 / p_{n}$. The optimization problem can be described as 
Optimization Problem SMAP (Sequentially Minimal Axis Problem):

$$
\begin{array}{ll}
\operatorname{minimize} & 1 / p_{i} \\
\text { subject to } & A^{T} p \leq \leq 0, B^{T} p \leq \leq \mathbf{1},
\end{array}
$$

where $i=1,2, \ldots, n, p=\left[p_{1}, p_{2}, \ldots, p_{n}\right]^{T}$.

As the intersection of these $n$ hyper-pyramids $\mathcal{C}_{p}^{i}(i=$ $1,2, \ldots, n)$, that is $\bigcap_{i=1}^{n} \mathcal{C}_{p}^{i}$, must enclose the system reachable set. Therefore, the hyper-volume of $\bigcap_{i=0}^{n} \mathcal{C}_{p}^{i}$ is certainly not larger than the hyper-volume of $\mathcal{C}_{p}^{0}$, so that Optimization Problem SMAP can further reduce the bounding reachable set.

\subsection{Estimation Example}

In this section, we present an illustrative example to demonstrate the applicability of the proposed results. The most popular model for describing river pollution is, still now, the Streeter and Phelps model proposed in 1925. Our aim is to evaluate the water pollution situation in a certain area. The model is written as

$$
\begin{aligned}
& \dot{x}_{1}(t)=-k_{1} x_{1}(t)+\omega(t), \\
& \dot{x}_{2}(t)=k_{1} x_{1}(t)-k_{2} x_{2}(t),
\end{aligned}
$$

where $k_{1}>0$ is deoxygenation constant, $k_{2}>0$ is reoxygenation constant; $x_{1}(t)$ is the concentration of biodegradable matter contained in the water which is, for simplicity and by convention, expressed in terms of biochemical oxygen demand (BOD); $x_{2}(t)$ is the dissolved oxygen deficit (DOD) (measured in $[\mathrm{mg} / \mathrm{l}]$ ) which is the difference between the highest concentration of dissolved oxygen in water and the actual one; $\omega(t) \geq 0$ is the BOD discharge rate into the river from the environment. Since a unit of BOD is the concentration of biodegradable matter requiring one unit of oxygen to be degraded by bacteria, the same term $k_{1} x_{1}(t)$ appears in both state equations with an opposite sign. The term $-k_{2} x_{2}(t)$ in the second equation represents the diffusion of oxygen from the air into water at a rate proportional to the oxygen deficit [9]. The flow time $t$ is the time needed for each drop of water to reach the generic section starting from $t=0$. Thus, the Streeter and Phelps model is a typical positive linear system.

The model can be rewritten in the form of system (1) with

$$
A=\left[\begin{array}{rr}
-k_{1} & 0 \\
k_{1} & -k_{2}
\end{array}\right], B_{\omega}=\left[\begin{array}{l}
1 \\
0
\end{array}\right],
$$

where $A$ is a Metzler matrix. The reachable set represents the set composed of all the pairs $\left(x_{1}, x_{2}\right)$ generated at all $t \in[0,+\infty)$, that is, all the possible amounts of BOD and DOD in water under the external disturbance

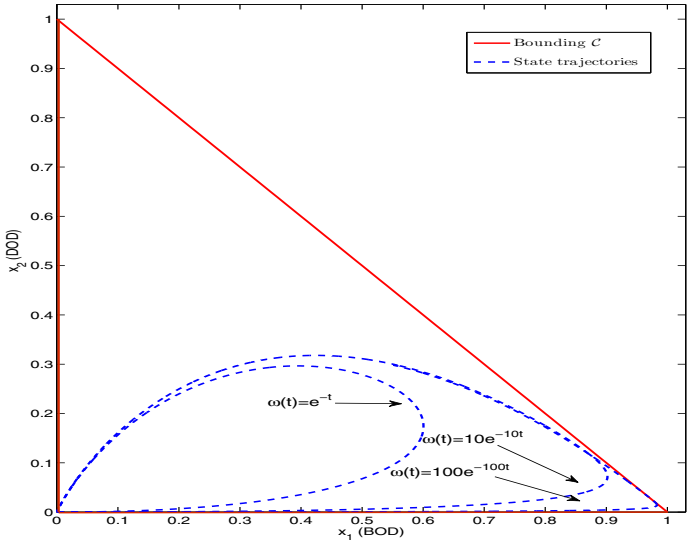

Fig. 1. $\omega(t)=c e^{-c t}, c=1,10,100,\left(\right.$ in $\left.\Omega_{1,1}\right)$.

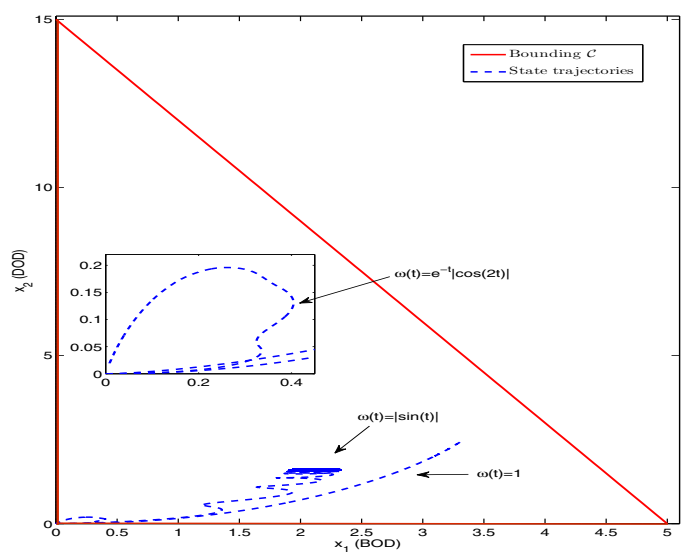

Fig. 2. $\omega(t)=e^{-t}|\cos (2 t)|,|\sin (t)|$, or 1 (in $\Omega_{\infty, 1}$ ).

$\omega(t)$. To illustrate the estimation, fix $k_{1}=0.3, k_{2}=0.4$. The unique optimal triangular region $\mathcal{C}_{p}$ obtained by Optimization Problem MHVP is given by $p=[1,1]^{T}$ which encloses all possible system states when $\omega \in \Omega_{1,1}$. Assuming the external disturbance $\omega(t)$, respectively, as $e^{-t}, 10 e^{-10 t}, 100 e^{-100 t}$ (these are also admissible disturbances belong to $\Omega_{1,1}$ since $\int_{0}^{+\infty} c e^{-c \cdot t} d t=1$, when $c$ equals to 1,10 , or 100), the trajectories of changes of BOD and DOD in the water body are shown in Figure 1, which reflect the changes of the oxygen uptake by bacteria and the amount of gaseous oxygen dissolved in a water sample. When $\omega(t)=100 e^{-100 t}$, it can be approximately taken as a lumped discharge present in section $t=0$ (if $\omega(t)$ equals a Dirac delta at $t=0$, then the point $(1,0)$ is reachable).

When $\omega \in \Omega_{\infty, 1}$, according to Remark 3, $\alpha$ appearing in Theorem 3 should be chosen within the interval $(0,0.3]$. By solving Optimization Problem MHVP, an optimal triangular region $\mathcal{C}_{p}$ is associated with $p=$ $[1 / 5,1 / 15]^{T}$ and $\alpha=0.2$. Taking $\omega(t)$ as $e^{-t}|\cos (2 t)|$, $\omega(t)=|\sin (t)|$, or even $\omega(t) \equiv 1$, changes of the amounts of BOD and DOD in the water body are shown in Figure 2. The area of $\mathcal{C}_{p}$ versus $\alpha$ in this case can be derived 


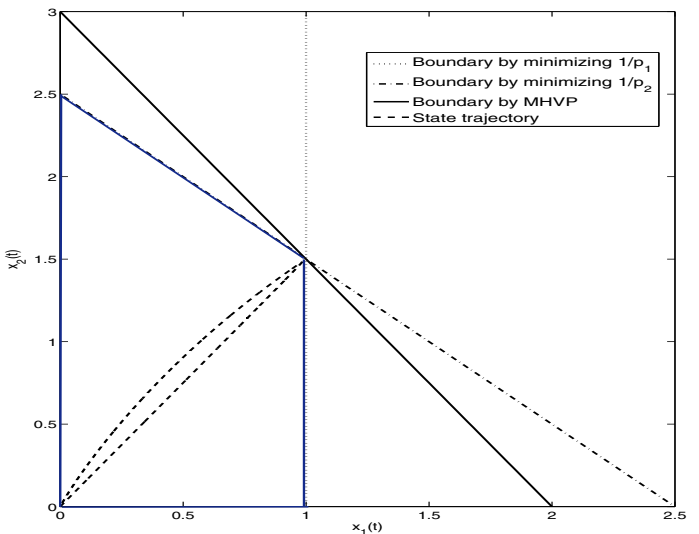

Fig. 3. System trajectory for $\omega(t)=100 e^{-100 t}$ and bounding hyper-pyramid.

as $0.15 /\left[\alpha^{2}(0.3-\alpha)\right]$. Its unique minimum is given by $\alpha=0.2$.

It should be noted that, in this example, the optimal positive vectors $p$ obtained respectively by Optimization Problems MHVP and SMAP are the same. Supposing now $B_{\omega}=[1,1.5]^{T}$, there are three optimal solutions for $p$, and they are $\left[1,0^{+}\right]^{T},[2 / 5,2 / 5]^{T}$, and $[1 / 2,1 / 3]^{T}$ obtained by minimizing $1 / p_{1}, 1 / p_{2}$, and the hyper-volume of $\mathcal{C}_{p}$, respectively. Three different bounding regions will hence be constructed (see Figure 3). Apparently, the intersection of these three regions, whose boundary is depicted with bold lines, is certainly bounding the system reachable set and has smaller area. In this example, the set consisting of all the feasible solutions for $p$ satisfying $A^{T} p \leq \leq 0$ and $B^{T} p \leq \leq \mathbf{1}$ can be shown as the shaded triangular area in Figure 4 . In this feasible region, the largest $p_{1}=1$ (that is, the smallest $1 / p_{1}$ ) is achieved in the corner point $\left(1,0^{+}\right)$, that is also the optimal $p_{1}$ obtained in Optimization Problems SMAP by minimizing $1 / p_{1}$ (the point $(1,0)$ is reachable with $\omega(t)$ equals a Dirac delta of unit strength acting at some $t>0)$. In other words, point $\left(1,0^{+}\right)$generates the vertical dotted line in Figure 3. The corner point $(2 / 5,2 / 5)$ with the largest $p_{2}=2 / 5$ in the feasible region corresponds to the dashdotted line in Figure 3. Notice that the optimization problem to find a smallest feasible hyper-pyramid is equivalent to solving maximization of a variable $c \triangleq p_{1} p_{2}$. The graph of $p_{1} p_{2}=c, c \in \mathbb{R}_{+}$represents a family of hyperbolas in the first orthant, and the optimal $c$ (denoted as $c_{\text {opt }}$ ) is achieved when the hyperbola tangents to the boundary to the right of the shaded triangular region. More precisely, in Figure 4, the point of tangency is $(1 / 2,1 / 3)$ and thus $c_{o p t}=1 / 6$, which corresponds to the solid line in Figure 3. There is an interesting fact that these three points fall on a line, $p_{1}+1.5 p_{2}=1$, which is the boundary of the region determined by $B^{T} p \leq \leq \mathbf{1}$. This is the reason why three boundary line segments in Figure 3 pass through the same point at $(1,1.5)$.

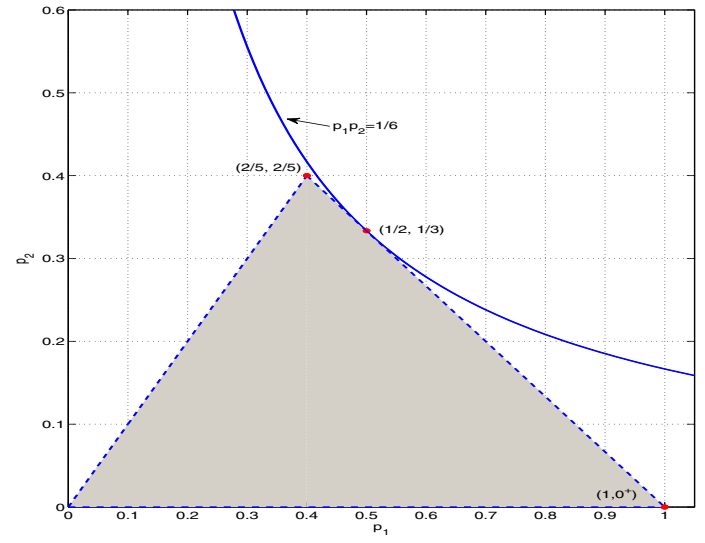

Fig. 4. Feasible solutions $p=\left[p_{1}, p_{2}\right]^{T}$ when $B_{\omega}=[1,1.5]^{T}$.

\section{State-feedback Synthesis}

In this section, the state-feedback synthesis problem, that is, the problem of finding a state-feedback controller gain will be considered so that the closed-loop system satisfies positivity and its reachable set can be restrained within a prescribed hyper-pyramid.

\subsection{Controller Design}

Consider a positive linear system with a zero initial state:

$$
\dot{x}(t)=A x(t)+B_{u} u(t)+B_{\omega} \omega(t)
$$

where $x(t) \in \overline{\mathbb{R}}_{+}^{n}, u(t) \in \mathbb{R}^{p}, \omega(t) \in \overline{\mathbb{R}}_{+}^{m}$ are the system state, the control input and an exogenous input signal, respectively, $A \in \mathbb{R}^{n \times n}$ is Metzler, $B_{u} \in \overline{\mathbb{R}}_{+}^{n \times p}$ and $B_{\omega} \in$ $\overline{\mathbb{R}}_{+}^{n \times m}$ are constant system matrices. Let $K \in \mathbb{R}^{p \times n}$, and suppose that $u(t)=K x(t)$, which yields the closed-loop system

$$
\dot{x}(t)=\left(A+B_{u} K\right) x(t)+B_{\omega} \omega(t) .
$$

Based on the analysis results described in Section 3, the following proposition gives conditions under which a state-feedback gain $K$ exists to guarantee a given hyperpyramid $\mathcal{C}_{p}$ containing all the states of system (14) for different classes of exogenous inputs.

Proposition 1 For a prescribed hyper-pyramid $\mathcal{C}_{p}$, if there exist a vector $p \in \mathbb{R}_{+}^{n}$, a scalar $\alpha>0$, and a statefeedback gain $K \in \mathbb{R}^{p \times n}$ satisfying that $A+B_{u} K$ is Metzler, and

$$
\begin{array}{r}
\text { if } \omega \in \Omega_{1,1},\left\{\begin{array}{r}
p^{T}\left(A+B_{u} K\right) \leq \leq 0 ; \\
B_{\omega}^{T} p \leq \leq \mathbf{1} ;
\end{array}\right. \\
\text { or, if } \omega \in \Omega_{\infty, 1},\left\{\begin{array}{r}
p^{T}\left(A+B_{u} K\right)+\alpha p^{T} \leq \leq 0 ; \\
B_{\omega}^{T} p \leq \leq \alpha \mathbf{1} .
\end{array}\right.
\end{array}
$$


then $\mathcal{C}_{p}$ contains the reachable set of the closed-loop system (14).

Since the hyper-pyramid $\mathcal{C}_{p}$ is prescribed, and hence the vector $p$ is known, (15) and (16) are linear with respect to variables $K$ and $\alpha$, their feasibility can be verified easily by linear programming.

On the other hand, if the existence of a state-feedback controller gain $K$, can be characterized according to Proposition 1 such that the states of the closed-loop system are enclosed by a known hyper-pyramid $\mathcal{C}_{p}=\{\xi \in$ $\left.\overline{\mathbb{R}}_{+}^{n} \mid p^{T} \xi \leq 1, p \in \overline{\mathbb{R}}_{+}^{n}\right\}$, one may attempt to further reduce the given hyper-pyramid. A natural way is to fix the feasible solution $K$, and solve (15) or (16) with the help of linear programming and convex optimization, which is easy to achieve by the following algorithm. Take Case (i) as an example, with Theorem 1 , for fixed $K$, solve the following optimization problem for $p=\left[p_{1}, p_{2}, \ldots, p_{n}\right]^{T}$.

$$
\begin{array}{ll}
\text { minimize } & \gamma \triangleq-\sum_{i=1}^{n} \log p_{i} \\
\text { subject to } & \left(A+B_{u} K\right)^{T} p \leq \leq 0, B_{u}^{T} p \leq \leq \mathbf{1} .
\end{array}
$$

Denote $\gamma^{(*)}$ as the minimum value of $\gamma$, and the corresponding solution $p$ to $\gamma^{(*)}$ is denoted by $p^{(*)}$. Since $K$ is the feasible solution of $(17)$, we conclude that the hypervolume of hyper-pyramid $\mathcal{C}_{p}^{(*)}$ corresponding to $\gamma^{(*)}$ and $p^{(*)}$ is not larger than that of $\mathcal{C}_{p}$. The sequence of $\gamma^{(*)}$ is thus non-increasing and bounded from below by zero. Hence, the parameter $\gamma$ can be optimized iteratively.

\subsection{Design Example}

In this example, we demonstrate the applicability of the proposed results on the controller design problem for system (13) with two classes of disturbance inputs. To this end, consider the positive linear system in (13) with system parameters given as follows [7]:

$$
A=\left[\begin{array}{ccc}
-2 & 1.3 & 1 \\
0.5 & -3 & 0.7 \\
2 & 1.5 & -2
\end{array}\right], B_{u}=\left[\begin{array}{cc}
1 & 0 \\
0 & 1 \\
1 & 0.5
\end{array}\right], B_{\omega}=\left[\begin{array}{l}
0.1 \\
0.2 \\
0.8
\end{array}\right]
$$

For a prescribed pyramid $\mathcal{C}_{p}$ with $p=\left[\begin{array}{l}1.5,1.5,0.9 \\ ]^{T}\end{array}\right.$ shown in Figure 5, part of state trajectory of the openloop system with $\omega(t)=10 e^{-10 t}\left(\omega \in \Omega_{1,1}\right)$ is outside pyramid $\mathcal{C}_{p}$. Through Proposition 1 , a state-feedback controller is designed with gain

$$
K=\left[\begin{array}{ccc}
-0.64 & -0.5 & -0.1 \\
-0.0013 & -0.78 & -0.51
\end{array}\right] \text {, }
$$

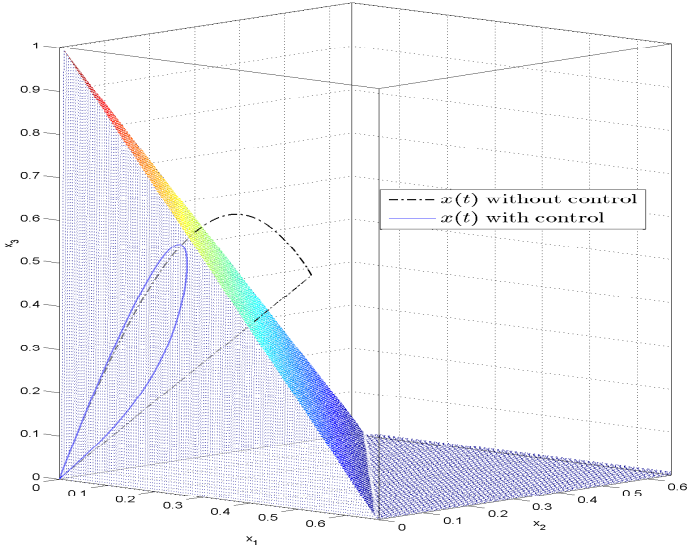

Fig. 5. System trajectory for $\omega(t)=10 e^{-10 t}$ and hyper-pyra$\operatorname{mid} \mathcal{C}_{p}$.

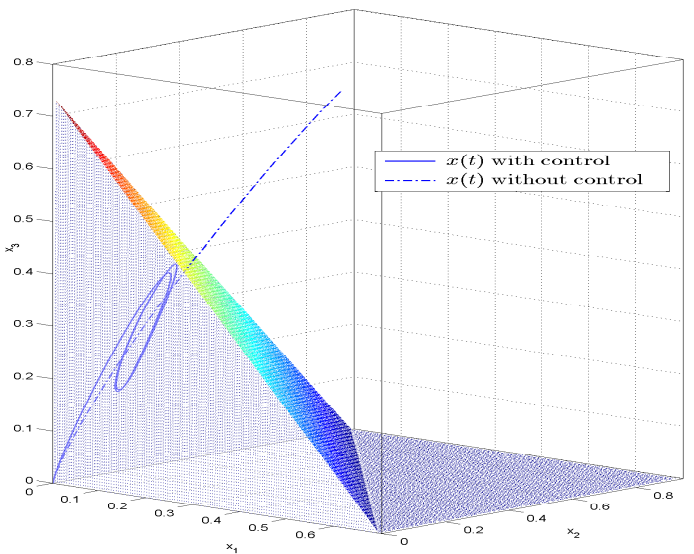

Fig. 6. System trajectory for $\omega(t)=|\sin (t)|$ and hyper-pyra$\operatorname{mid} \mathcal{C}_{p}$.

and then the closed-loop system matrix is given by

$$
A+B_{u} K=\left[\begin{array}{ccc}
-2.64 & 0.8 & 0.9 \\
0.4987 & -3.78 & 0.19 \\
1.3594 & 0.61 & -2.355
\end{array}\right]
$$

which is Metzler. It is apparent from Figure 5 that the state response of the closed-loop system is restrained within $\mathcal{C}_{p}$.

For another pyramid $\mathcal{C}_{p}$ with $p=[0.2,0.2,0.3]^{T}$, the state trajectory of the open-loop system with $\omega(t)=$ $|\sin (t)|\left(\omega \in \Omega_{\infty, 1}\right)$ goes out of the hyper-pyramid. By applying Proposition 1, the closed-loop system trajectories are restrained within $\mathcal{C}_{p}$ via the same controller designed above, see Figure 6.

\section{Conclusions}

This paper has investigated the analysis and synthesis of the reachable set bounding problem for positive 
linear systems with the zero initial state and a specific disturbance. In the analysis aspect, the Lyapunov approach has been applied to find a hyper-pyramid that bounds the set of the reachable system states, subjec$\mathrm{t}$ to disturbances whose $(1,1)$-norm or $(\infty, 1)$-norm is bounded by a prescribed constant. Two convex optimization problems (with an adjustable parameter) have been proposed to minimize the hyper-volume of the bounding hyper-pyramid. In the synthesis aspect, a state feedback controller has been designed which guarantees the reachable set of the closed-loop system bounded by a given hyper-pyramid. The desired controllers can be constructed through an iterative linear programming method.

\section{Acknowledgment}

This work was partially supported by the Research Grants Council of Hong Kong under project GRF HKU 7137/13E, the National Natural Science Foundation of China under Grants 61573188, the Fundamental Research Funds for the Central Universities No. 30915011327, and the Natural Science Foundation of Jiangsu Province, China (Grant No. BK20151492).

\section{References}

[1] J. Abegor, K. Nagpal, and K. Poolla. A linear matrix inequality approach to peak-to-peak gain minimization. Int. J. Control, 72:374-384, 1996.

[2] S. Boyd, L. El Ghaoui, E. Feron, and V. Balakrishnan. Linear Matrix Inequalities in Systems and Control Theory. SIAM, Philadelphia, PA, 1994

[3] C. Briat. Robust stability and stabilization of uncertain linear positive systems via integral linear constraints: $l_{1}$-gain and $L_{\infty}$-gain characterization. Int. J. Robust $\& 3$ Nonlinear Control, 23(7):1932-1954, 2013.

[4] J. S. Carter and A. Champanerkar. A geometric method to compute some elementary integrals. http://arxiv.org/pdf/math/0608722.

[5] H. Chen, J. Cheng, S. Zhong, J. Yang, and W. Kang. Improved results on reachable set bounding for linear systems with discrete and distributed delays. Chaos, Solitons \& Fractals, Article Number: 145, DOI: 10.1186/s13662-0150479-6, 2015.

[6] X. Chen, J. Lam, P. Li, and Z. Shu. $\ell_{1}$-induced norm and controller synthesis of positive systems. Automatica, 49(5):1377-1385, 2013

[7] X. Chen, J. Lam, P. Li, and Z. Shu. $\ell_{1}$-induced performance analysis and sparse controller synthesis for interval positive systems. In Proceedings of the World Congress on Engineering, volume 1, London, U.K., July 2013.

[8] C. Durieu, E. Walter, and B. Polyak. Multi-input multioutput ellipsoidal state bounding. J. Optim. Theory Appl., 111(2):273-303, 2001

[9] L. Farina and S. Rinaldi. Positive Linear Systems: Theory and Applications. Wiley, New York, 2000.

[10] A. L. Fradkov. Duality theorems for certain nonconvex extremal problems. Siberian Mathematical Journal, 14(2):357-383, 1973.
[11] E. Fridman and U. Shaked. On reachable sets for linear systems with delay and bounded peak inputs. IEEE Trans. Automat. Control, 39:2005-2010, 2003.

[12] E. Goncharova and A. Ovseevich. Small-time reachable sets of linear systems with integral control constraints: birth of the shape of a reachable set. J. Optim. Theory Appl., 168(2):615624, 2016.

[13] W. M. Haddad and V. Chellaboina. Stability and dissipativity theory for nonnegative dynamical systems: a unified analysis framework for biological and physiological systems. Nonlinear Analysis: Real World Applications, 6:3565, 2005.

[14] W. M. Haddad, V. Chellaboina, and T. Rajpurohit. Dissipativity theory for nonnegative and compartmental dynamical systems with time delay. IEEE Trans. Automat. Control, 49(5):747-751, 2004.

[15] T. Hu, Z. Lin, and B. Chen. An analysis and design method for linear systems subject to actuator saturation and disturbance. Automatica, 38:351-359, 2002.

[16] U. T. Jönsson. A lecture on the S-procedure. Lecture Note at the Royal Institute of Technology, Sweden, 2001, Available: http://www.math.kth.se/ulfj/5B5746/Lecture.ps.

[17] W. Kang and S. Zhong. New results on reachable set estimation and controller design for linear systems with mixed delays via triple integral functionals. Discrete Dynamics in Nature and Society, Article Number: 935920, 2015.

[18] J.-H. Kim. Improved ellipsoidal bound of reachable sets for time-delayed linear systems with disturbances. Automatica, 44:2940-2943, 2008.

[19] X. Liu and C. Dang. Stability analysis of positive switched linear systems with delays. IEEE Trans. Automat. Control, 56(7):1684-1690, 2011.

[20] X. Liu, W. Yu, and L. Wang. Stability analysis for continuous-time positive systems with time-varying delays. IEEE Trans. Automat. Control, 55(4):1024-1028, 2010.

[21] P. T. Nam, P. N. Pathirana, and H. Trinh. Reachable set bounding for nonlinear perturbed time-delay systems: The smallest bound. Applied Mathematics Letters, 43:68-71, 2015.

[22] S. Tarbouriech, G. Garcia, and J. Gomes da Silva. Robust stability of uncertain polytopic linear time-delay systems with saturating inputs: An LMI approach. Computer \&5 Electrical Engineering, 28(3):157-169, 2002.

[23] H. Trinh, P. T. Nam, P. N. Pathirana, and H. P. Le. On backwards and forwards reachable sets bounding for perturbed time-delay systems. Applied Mathematics and Computation, 269:664-673, 2015.

[24] C. Wang and T. Huang. Static output feedback control for positive linear continuous-time systems. Int. J. Robust 8 Nonlinear Control, 23(14):1537-1544, 2013.

[25] M. Xiang and Z. Xiang. Stability, $L_{1}$-gain and control synthesis for positive switched systems with time-varying delay. Nonlinear Analysis Hybrid Systems, 8:9-17, 2013.

[26] V. A. Yakubovich. S-procedure in nonliear control theory. Vestnik Leningrad University, pages 62-77, 1971.

[27] B. Zhang, J. Lam, and S. Xu. Reachable set estimation and controller design for distributed delay systems with bounded disturbances. Journal of the Franklin Institute, 351:30683088, 2014.

[28] Z. Zuo, Z. Wang, Y. Chen, and Y. Wang. A non-ellipsoidal reachable set estimation for uncertain neural networks with time-varying delay. Communications in Nonlinear Science and Numerical Simulation, 19(4):1097-1106, 2014. 